\title{
Expression of Concern: Selective impairment of insulin signalling in the hypothalamus of obese Zucker rats
}

\author{
J. B. C. Carvalheira ${ }^{1}$ - E. B. Ribeiro ${ }^{2}$ E. P. Araújo ${ }^{1}$ - R. B. Guimarães ${ }^{2} \cdot$ M. M. Telles ${ }^{2}$. $^{2}$ \\ M. Torsoni ${ }^{1}$ - J. A. R. Gontijo ${ }^{1}$ - L. A. Velloso ${ }^{1}$ M. J. A. Saad ${ }^{1}$
}

(C) Springer-Verlag GmbH Germany 2017

\section{Expression of Concern relating to Diabetologia \\ DOI 10.1007/s00125-003-1246-x}

On the basis of the recommendation of the EASD's Scientific Integrity Panel, the Editor-in-Chief is issuing this expression of concern to alert readers to questions about the reliability of some of the data in the article cited above. The concerns include, but are not limited to:

(1) the use of different membranes for the p-ERK1 and -2 and ERK1 and -2 blots shown in Fig. 6b. The figure legend implies, although does not explicitly state, that the same membrane is shown in the upper and lower images stripped and reblotted: 'Stripped membranes were reblotted with anti-AKT and anti-ERK antibodies'.

In line with guidelines issued by the Committee on Publication Ethics (COPE), the journal informed the corresponding author, Mario Saad, of this concern. Dr Saad has confirmed that different membranes could indeed have been used in Fig. $6 \mathrm{~b}$ as the authors selected the clearest and most representative blots from ten experiments.

The online version of the original article can be found at http://dx.doi.org/ 10.1007/s00125-003-1246-x

M. J. A. Saad

msaad@fcm.unicamp.br

1 Departamento de Clínica Médica, FCM, Universidade Estadual de Campinas (UNICAMP), Campinas, Brazil

2 Departamento de Fisiologia, Universidade Federal de São Paulo (UNIFESP), São Paulo, SP, Brazil
Fig. 6b

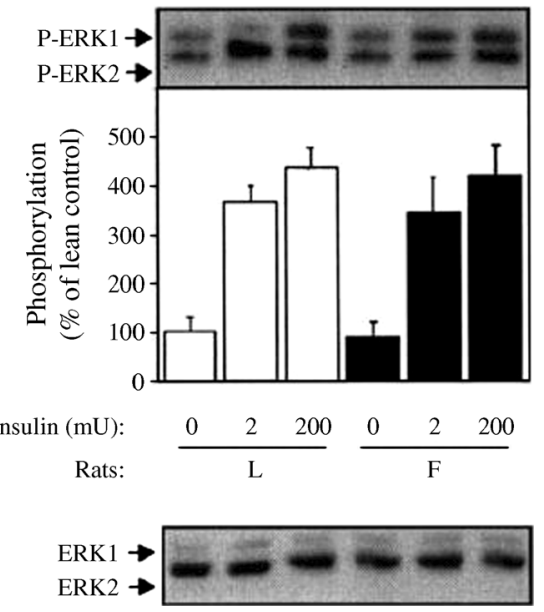

(2) representative images for loading controls are absent in several figures. The journal accepts some responsibility for this omission, as this was not picked up during the peer-review process.

The University of Campinas (São Paulo, Brazil) is undertaking an institutional investigation into the work carried out by members of this group. The results of this investigation are not yet available, thus this expression of concern is being issued in the interim to alert readers to exercise caution when interpreting the content and conclusions of this article. This expression of concern will remain in place until such a time as further evidence is available. 